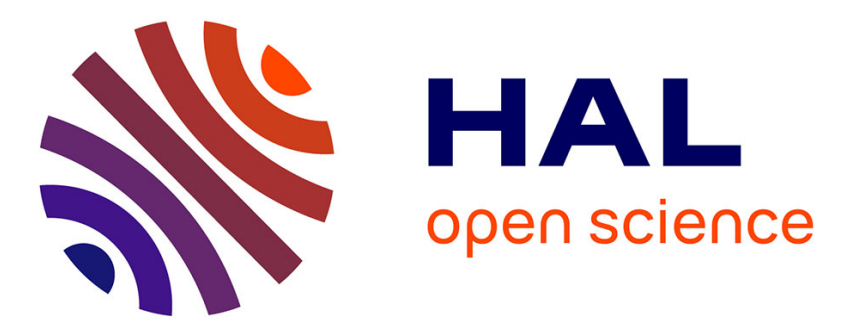

\title{
"Fast" and "slow" pressure waves electrically induced by nonlinear coupling in Biot-type porous medium saturated by a nematic liquid crystal
}

\author{
Giuseppe Rosi, Luca Placidi, Francesco Dell'Isola
}

\section{- To cite this version:}

Giuseppe Rosi, Luca Placidi, Francesco Dell'Isola. "Fast" and "slow" pressure waves electrically induced by nonlinear coupling in Biot-type porous medium saturated by a nematic liquid crystal. Zeitschrift für Angewandte Mathematik und Physik, 2017, 68 (2), pp.51. 10.1007/s00033-017-07957. hal-01574781

\author{
HAL Id: hal-01574781 \\ https://hal.science/hal-01574781
}

Submitted on 18 Aug 2017

HAL is a multi-disciplinary open access archive for the deposit and dissemination of scientific research documents, whether they are published or not. The documents may come from teaching and research institutions in France or abroad, or from public or private research centers.
L'archive ouverte pluridisciplinaire HAL, est destinée au dépôt et à la diffusion de documents scientifiques de niveau recherche, publiés ou non, émanant des établissements d'enseignement et de recherche français ou étrangers, des laboratoires publics ou privés. 


\title{
"Fast" and "slow" pressure waves electrically induced by nonlinear coupling in Biot-type porous medium saturated by a nematic liquid crystal
}

\author{
Giuseppe Rosi, Luca Placidio and Francesco dell'Isola
}

\begin{abstract}
In this paper, it is proposed a model for deformable porous media saturated by compressible nematic liquid crystal subjected to slowly varying electric fields. from a mechanical point of view, we assume that such a system can be described by means of a Biot-type model and that the mechanical action of the NLC on the solid matrix can be modeled by means of a suitable modification of Biot constitutive equations for pore pressure only. The nonlinear nature of NLCs and the presence of bifurcations make the analysis particularly challenging. We prove that suitable electrical stimulus applied on the NLC specimen may induce both type of Biot waves, fast and slow, along with shear waves in the porous matrix. This effect may be of use when one may wish to damp mechanically induced pressure waves using Darcy dissipation.
\end{abstract}

\section{Introduction}

The same expression "Liquid Crystals" may seem an oxymoron. The reader is referred to the beautiful book by De Gennes and Prost [1] for an elegant clarification of those which finally are only apparent contradictions. We agree with De Gennes and Prost in believing that a more proper name is "fluid showing mesomorphic phases". The continuum description of Liquid Crystals requires the choice of suitable constitutive equations which are intermediate between those needed for solids and fluids. Using the nomenclature proposed in Eringen [2], Liquid Crystals are micropolar continua. They are mostly known for their electro-optic properties, as Liquid Crystal Displays (LCDs) are everyday more common. The phenomenon that is exploited for building these devices is the coupling between the orientation of the molecules of the crystal with an electromagnetic fields. This reorientation changes the refractive index of the crystals and thus influences how the light is propagating through it. However, since Liquid Crystals usually behave as incompressible fluids, very few researchers investigated the acousto-optic properties of these materials, while some experiments conducted by Kim and Patel [3] show without any doubt that also these interactions may occur so that further theoretical and experimental investigations on this phenomenon are needed. The nonlinear nature of nematic liquid crystals (NLCs) and the presence of bifurcations paths in director field configuration when varying applied electric fields make the analysis of dynamic phenomena particularly challenging.

Nematic liquid crystals (NLC) are usually modeled as incompressible fluids. When acoustic compression waves in NLC are considered, only their influence on electromagnetic fields propagation and optics is usually taken into account. On the other hand, it is possible to find experimental evidence of

\footnotetext{
Liquid Crystals are beautiful and mysterious
}

P.G. De Gennes. 
acoustic waves generation induced by electromagnetic signals, and of the related nonlinear constitutive equation for the pressure that is necessary for describing this experimental evidence. Besides, the coupling phenomena occurring in complex systems constituted by (i) highly porous matrices, (ii) saturating compressible NLC, (iii) electric devices inducing quasi-static electric fields, have attracted the attention of many investigators.

Even if it is less known than the more studied electro-optic effect, the acousto-optic effect has been object of some researches. In particular some authors [4-8] have established that the reorientation of the molecules of a NLC can be induced by an external acoustic wave, by providing a mathematical model and clear experimental evidence $[9,10]$. They propose to use an interaction energy proportional to the nematic director $\mathbf{n}$ and to the gradient of the density $\varrho$, in fact modeling the NLC as a second gradient fluid [11]. More recently, Virga $[12,13]$ proposed a more complete second gradient model (as they include also the gradient of the pressure in the coupling term) with the aim of explaining the same phenomenon. Although these models have a good agreement with some experimental evidence, they have some drawbacks. In particular, they are not able to catch the converse phenomenon of measuring an acoustic signal generated by the reorientation of the molecules, phenomenon which was actually observed in [3].

Differently from what done by aforementioned authors, in this work, by following [14] we adopt a model for a compressible NLC in which the spatial gradient of the director field $\mathbf{n}$ is coupled with the mass density $\varrho$. Few words are needed to justify the ansatz which is at the base of [14]: when the directors of the nematic fluid are all parallel, then the molecules of the fluid are all packed in the most "ordered way". Therefore in this configuration the density of the liquid crystal attains its maximum value. When close material particles of the liquid crystals have directors oriented in different directions, once fixed the liquid pressure, the density varies depending on the assumed directors' configuration. This occurs because the molecules of the fluid crystal, in the presence of gradients of orientation, are more "loosely packed" and consequently the density is lower.

In the present paper we consider a solid deformable porous matrix with interconnected pores, saturated with a nematic liquid crystal. From a mechanical point of view, we assume that the system under consideration can be described, for what concerns the solid-fluid interaction phenomena, by means of a Biot-type model and we limit ourselves to the case of a solid matrix that has a negligible electric susceptibility and high porosity. We assume the validity of balance equations for the solid and fluid phases together with balance of torques for NLC, and we complete the modeling process by suitably assuming general enough constitutive equations. The reason is that, when considering a continuum model for porous media saturated by liquid phases, there is no "a priori" reason for assuming that any constituent of considered continuum has to be incompressible or that no compression (acoustic) or shear waves can arise as a secondary effect of coupling phenomena and in particular as a consequence of the propagation of coupled electromagnetic-orientation waves.

While we still consider that electromagnetic-nematic evolution is quasi-static (and we still use standard Frank energy density), we include some mechanical inertial effects when varying in time the applied external voltage. These choice will allow us to observe numerically the initiation of coupled acoustic waves.

\section{A continuum model for a porous matrix saturated with nematic liquid crystals}

While there is no difficulty in formulating the general modeling framework for considered phenomena in the more general case of three dimensional systems, for sake of simplicity, we limit ourselves to present balance equations valid for two dimensional systems. The main features of the phenomena that we intend to model are still captured when this simplification is applicable. Indeed, we will consider the case of a thin cell constituted by a porous deformable matrix infused with a NLC and immersed in an externally controlled quasi-static electric field so that we are allowed to consider a reduced planar problem. 


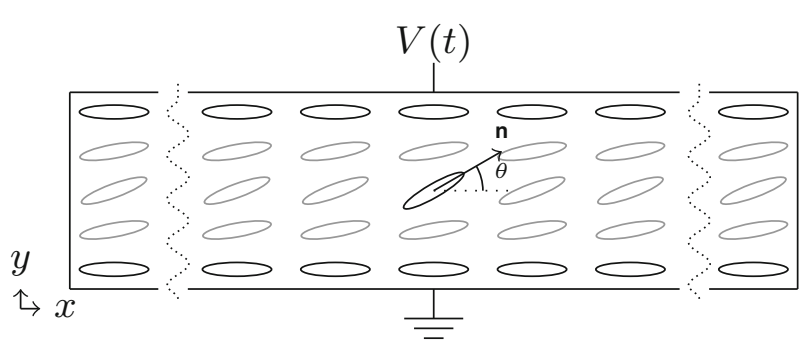

FiG. 1. Geometry of the nematic cell

\subsection{Description of the considered physical configuration}

In the present paper, we want to study the behavior of a bi-dimensional resonator having a rectangular shape with very small aspect ratio, constituted by (i) two conductive plates and time varying electric potential, (ii) a porous matrix having high porosity and low electric susceptibility and (iii) a nematic liquid crystal saturating the pores (Fig. 1). We suppose no anchoring interaction between the NLC and inner surface of the pores, so that in particular the porous matrix does not interfere with the phase transitions of the NLC.

Although this conceived resonator has a more general nature of the one experimentally studied in [3] and theoretically described in [14], our investigation proves that the main features observed or described by aforementioned works still can be observed as a particular case, where the elastic moduli and mass density of porous matrix are neglected, or where suitable coupling coefficients will be set to zero. However, interesting new coupled resonating eigenmodes are forecast by our modeling procedure and interesting physical phenomena are described, which could find interesting applications.

\subsection{Kinematics of the planar problem}

Let us consider a Lagrangian configuration for a deformable porous matrix saturated by a compressible nematic liquid crystal, and let us denote by $\mathbf{r}$ the generic point of the reference space which refers to the solid matrix material points. The state of the system is characterized by the following time varying spatial fields: the nematic director $\mathbf{n}(\mathbf{r}, t)$, the electric potential $\phi(\mathbf{r}, t)$, the displacement of the solid matrix $\mathbf{u}(\mathbf{r}, t)$ and the relative displacement of the NLC with respect to the solid matrix $\mathbf{w}(\mathbf{r}, t)$. For making the notation lighter the dependence on space and time will be omitted when this will not cause misunderstanding. As for NLC, the states corresponding to $\mathbf{n}$ and $\mathbf{- n}$ are indistinguishable and the molecules are symmetric with respect to rotations about their axis [15], the correct kinematical descriptor of the nematic molecules results to be the tensor $\mathbf{n} \otimes \mathbf{n}$. In the considered $2 \mathrm{D}$ cell we have that $\mathbf{n}=\mathbf{n}(\theta):=\cos \theta \mathbf{e}_{1}+\sin \theta \mathbf{e}_{2}$ an it will be of use, in the following calculations, the introduction of the vector $* \mathbf{n}(\theta)=-\sin \theta \mathbf{e}_{1}+\cos \theta \mathbf{e}_{2}$, i.e., of the $\pi / 2$ anticlockwise rotation of $\mathbf{n}(\theta)$.

The starting point of our modeling procedure will be the well-established static theory of Nematics, as presented in $[1,15]$, and the classic theory of poroelasticity presented in [16-18]. In fact the NLC will be treated as a compressible fluid, with the addition of the constitutive equation which takes into account for the coupling between the distortion of the nematic field, the density of the liquid crystal and the deformation of the porous matrix. The vector field $\mathbf{n}$ models the pattern of relative alignment of nematic molecules in a given configuration. When this alignment field is distorted from a spatially uniform configuration, it exhibits an elastic-like behavior, since the field tends to return into the initial order when the alignment distortion vanishes. Moreover, the molecules tend to interact with external 
applied electric fields and tend to be aligned, in the case of positive uniaxial nematics, to such electric field.

\subsection{Constitutive equation for compressible NLCs confined in porous media}

As discussed in the introduction, NLCs cannot be modeled by means of a constitutive equation for pressure in which only the liquid mass density appears as an independent variable. Indeed, because of the microstructure of NLCs, their constitutive equations must involve the deformation gradient together with all the deformation measures which can be obtained combining to it also $\mathbf{n}$ and $\nabla \mathbf{n}$. In formulas we have that for a pure NLC the following constitutive equation can be considered:

$$
\frac{\varrho_{f}-\varrho_{0}}{\varrho_{0}}=\frac{1}{M}\left(p-p_{0}\right)-s(\mathbf{n}, \nabla \mathbf{n}) \text {. }
$$

In Eq. (1), $\varrho_{f}$ and $p$ are the pure NLC mass density and pressure, respectively, $\varrho_{0}$ and $p_{0}$ are suitable reference mass density and pressure respectively, $M$ is the NLC bulk modulus. Particular attention must be given to the term $s(\mathbf{n}, \nabla \mathbf{n})$, that is responsible for a mass density variation relative to $\varrho_{0}$, at constant pressure, induced by the distortion of the director field $\mathbf{n}$. Among the different possible definitions, we choose the following:

$$
s(\mathbf{n}, \nabla \mathbf{n})=\frac{1}{2} \delta|\nabla \cdot \mathbf{n}|^{2}=\frac{1}{2} \delta|\nabla \theta \cdot * \mathbf{n}|^{2},
$$

where $\delta$ is a suitable dilatational coefficient. As it can be easily checked, the positive definiteness of $s$ takes into account the cited phenomenon that ordered molecules are better packed. Equation (2) has the same form as Frank free energy, see e.g. [15] for details, in one constant approximation because we estimate the only relevant contribution to dilation comes from the splay term, that is related to the normal gradient.

When a compressible NLC is confined in a deformable porous matrix, saturating all its interconnected pores, Eq. (2) can be used to get a constitutive equation for the quantity that Biot calls pore pressure. The conservation of the mass for a fluid saturating a porous matrix reads

$$
\nabla \cdot \mathbf{w}+\frac{\varrho_{f}-\varrho_{0}}{\varrho_{0}}+\alpha_{B} \nabla \cdot \mathbf{u}=0
$$

where $\alpha_{B}$ is the Biot-Willis coefficient $[16,17]$. Equation (1) now reads

$$
\nabla \cdot \mathbf{w}=-\frac{1}{M} p_{f}+s(\mathbf{n}, \nabla \mathbf{n})-\alpha_{B} \nabla \cdot \mathbf{u}
$$

where $p_{f}=\left(p-p_{0}\right)$ is the pore pressure

\subsection{Balance equations}

The balance equation of torques for the NLC, under the assumption of absence of sources related to the coupling with deformation phenomena occurring into the fluid saturated porous matrix, is :

$$
\kappa_{\mathrm{F}} \Delta \theta+\varepsilon_{a}(\nabla \phi \cdot \mathbf{n}(\theta))(\nabla \phi \cdot * \mathbf{n}(\theta))=0,
$$

where $\nabla$ is the gradient operator, $\Delta$ is the Laplacian operator, $\kappa_{\mathrm{F}}$ is the Frank Elastic coefficient, $\varepsilon_{a}$ is the dielectric permittivity and $\phi$ is the electric potential. The boundary condition to be used when the nematic orientation is not subjected to strong anchoring is

$$
\kappa_{\mathrm{F}} \nabla \theta \cdot \mathbf{m}_{\partial \mathscr{C}}=\mu_{\partial \mathscr{C}},
$$


where $\mu_{\partial \mathscr{C}}$ is the intensity of the specific couple applied to the boundary of the NLC cell and $\mathbf{m}_{\partial \mathscr{C}}$ is the unit normal to the boundary. The equation of equilibrium that involves the electric field reduces to the well-known conservation of the charge

$$
\nabla \cdot(\mathbf{P} \nabla \phi)=0, \quad \mathbf{P}=\varepsilon_{v} \mathbf{I}+\varepsilon_{a}(\mathbf{n} \otimes \mathbf{n})
$$

and at the boundary the condition of absence of charge sources reads

$$
(\mathbf{P} \nabla \phi) \cdot \mathbf{m}_{\partial \mathscr{C}}=\left(\mathbf{P}_{\text {out }} \nabla \phi_{\text {out }}\right) \cdot \mathbf{m}_{\partial \mathscr{C}}
$$

where $\mathbf{P}_{\text {out }}$ and $\phi_{\text {out }}$ are, respectively, the permittivity and the electric potential field on the outer side of the boundary of the NLC cell.

The balance of linear momentum for solid and liquid constituents in the presence of pore pressure micro interaction reads $[16,17]$

$$
\begin{array}{r}
\rho_{a v} \frac{\partial^{2}}{\partial t^{2}} \mathbf{u}+\rho_{f} \frac{\partial^{2}}{\partial t^{2}} \mathbf{w}-\nabla \cdot \boldsymbol{\sigma}=0 \\
\rho_{f} \frac{\partial^{2}}{\partial t^{2}} \mathbf{u}+\frac{\tau}{\varepsilon_{p}} \rho_{f} \frac{\partial^{2}}{\partial t^{2}} \mathbf{w}+\nabla p_{f}=0
\end{array}
$$

where we have used the notation specified in Table 1. As done in Biot we manipulate Eqs. (8) and (9) by introducing the following decomposition

$$
\boldsymbol{\sigma}=\boldsymbol{\sigma}_{d r}-\alpha_{B} p_{f} \mathbf{I}
$$

and by using the modified expression of the pore pressure, Eq. (3). The final result is

$$
\begin{array}{r}
\left(\rho_{a v}-\frac{\rho_{f} \varepsilon_{p}}{\tau}\right) \frac{\partial^{2}}{\partial t^{2}} \mathbf{u}-\nabla \cdot\left(\boldsymbol{\sigma}_{d r}\right)=\left(\frac{\varepsilon_{p}}{\tau}-\alpha_{B}\right) \nabla p_{f} \\
\Delta p_{f}-\frac{\tau \rho_{f}}{M \varepsilon_{p}} \frac{\partial^{2}}{\partial t^{2}} p_{f}=-\rho_{f}\left(1-\alpha_{B} \frac{\tau}{\varepsilon_{p}}\right) \frac{\partial^{2}}{\partial t^{2}}(\nabla \cdot \mathbf{u})-\frac{\tau \rho_{f}}{\varepsilon_{p}} \frac{\partial^{2}}{\partial t^{2}} s
\end{array}
$$

Which in the case of $\tau=1$ reduces to

$$
\begin{array}{r}
\rho_{d r} \frac{\partial^{2}}{\partial t^{2}} \mathbf{u}-\nabla \cdot \boldsymbol{\sigma}_{d r}=\left(\varepsilon_{p}-\alpha_{B}\right) \nabla p_{f} \\
\Delta p_{f}-\frac{1}{c_{0}^{2}} \frac{\partial^{2}}{\partial t^{2}} p_{f}=-\frac{\rho_{f}}{\varepsilon_{p}}\left(\varepsilon_{p}-\alpha_{B}\right) \frac{\partial^{2}}{\partial t^{2}}(\nabla \cdot \mathbf{u})-\frac{\rho_{f}}{\varepsilon_{p}} \frac{\partial^{2}}{\partial t^{2}} s
\end{array}
$$

where $\rho_{d r}$ is defined in Table 1 and is the density of the drained porous material. Finally, since we are considering an homogeneous elastic solid matrix, we have that $\nabla \cdot \boldsymbol{\sigma}_{d r}=(\lambda+2 \mu) \nabla \nabla \cdot \mathbf{u}-\mu \nabla \times \nabla \times \mathbf{u}$,

TABLE 1. Main parameters and symbols

\begin{tabular}{ll}
\hline Quantity & Description \\
\hline $\mathbf{u}$ & Displacement of the solid matrix \\
$\mathbf{w}$ & Displacement of the fluid with respect to the solid matrix \\
$\rho_{f}$ & Total stress tensor \\
$\rho_{a v}=\rho_{d r}+\varepsilon_{p} \rho_{f}$ & Fluid density \\
$\mu_{f}$ & Average density \\
$\kappa$ & Fluid viscosity \\
$\varepsilon_{p}$ & Permeability \\
$\tau$ & Porosity \\
$p_{f}$ & Tortuosity \\
$\varepsilon_{v}, \varepsilon_{n}$ & Pore pressure \\
\hline
\end{tabular}


and the following final form for the balance equations is obtained

$$
\begin{gathered}
\rho_{d r} \frac{\partial^{2}}{\partial t^{2}} \mathbf{u}-(\lambda+2 \mu) \nabla \nabla \cdot \mathbf{u}+\mu \nabla \times \nabla \times \mathbf{u}=\left(\varepsilon_{p}-\alpha_{B}\right) \nabla p_{f} \\
\Delta p_{f}-\frac{1}{c_{0}^{2}} \frac{\partial^{2}}{\partial t^{2}} p_{f}=-\varepsilon_{p} \rho_{f}\left(\varepsilon_{p}-\alpha_{B}\right) \frac{\partial^{2}}{\partial t^{2}}(\nabla \cdot \mathbf{u})-\frac{\rho_{f}}{\varepsilon_{p}} \frac{\partial^{2}}{\partial t^{2}} s
\end{gathered}
$$

where we introduced the phase velocity of pressure waves as

$$
c_{0}=\sqrt{\frac{\varepsilon_{p} M}{\rho_{f}}}
$$

If we impose that the boundary $\partial \mathscr{C}$ of the porous matrix is fixed and impermeable for the NLC, then the following boundary conditions need to be considered

$$
\begin{array}{r}
\mathbf{u}=0, \\
\nabla p_{f} \cdot \mathbf{m}_{\partial \mathscr{C}}=0
\end{array}
$$

\subsection{Resume of obtained balance equations and boundary conditions}

The system of Partial Differential Equations (PDEs) is given by (4), (6), (12) and (13)

$$
\begin{array}{r}
\kappa_{\mathrm{F}} \Delta \theta+\varepsilon_{a}(\nabla \phi \cdot \mathbf{n}(\theta))(\nabla \phi \cdot * \mathbf{n}(\theta))=0 \\
\nabla \cdot(\mathbf{P} \nabla \phi)=0 \\
\rho_{d r} \frac{\partial^{2}}{\partial t^{2}} \mathbf{u}-(\lambda+2 \mu) \nabla \nabla \cdot \mathbf{u}+\mu \nabla \times \nabla \times \mathbf{u}=\left(\varepsilon_{p}-\alpha_{B}\right) \nabla p_{f} \\
\Delta p_{f}-\frac{1}{c_{0}^{2}} \frac{\partial^{2}}{\partial t^{2}} p_{f}=-\varepsilon_{p} \rho_{f}\left(\varepsilon_{p}-\alpha_{B}\right) \frac{\partial^{2}}{\partial t^{2}}(\nabla \cdot \mathbf{u})-\frac{\rho_{f}}{\varepsilon_{p}} \frac{\partial^{2}}{\partial t^{2}} s
\end{array}
$$

where we recall that $s$, as defined in Eq. (2), is responsible for the coupling between the directors field and the density of the fluid. The boundary conditions are given by (5), (7), (14) and (15):

$$
\begin{array}{r}
\kappa_{\mathrm{F}} \nabla \theta \cdot \mathbf{m}_{\partial \mathscr{C}}=\mu_{\partial \mathscr{C}}, \\
(\mathbf{P} \nabla \phi) \cdot \mathbf{m}_{\partial \mathscr{C}}=\left(\mathbf{P}_{\text {out }} \nabla \phi_{\text {out }}\right) \cdot \mathbf{m}_{\partial \mathscr{C}} \\
\mathbf{u}=0, \\
\nabla p_{f} \cdot \mathbf{m}_{\partial \mathscr{C}}=0 .
\end{array}
$$

\subsection{Coupled vibrations of considered poro-nematic cell}

In order to better understand which are the phenomena involved, and the nature of the coupling between electro-nematic equations and poroelastic equations, we can decompose the displacement field using the well-known Helmholtz decomposition

$$
\mathbf{u}=\nabla \varphi+\nabla \times \mathbf{U}
$$

where $\varphi$ is a scalar potential and $\mathbf{U}$ is a vector potential. In order to have a uniquely related field $\mathbf{u}$ to its potentials $\varphi$ and $\mathbf{U}$, one has also to add the following additional condition

$$
\nabla \cdot \mathbf{U}=0 .
$$


Using Eq. (24) into Eqs. (18) and (19), and using the well-known identities $\nabla \cdot(\nabla \times \mathbf{U})=0$ and $\nabla \times \nabla \phi=0$ we obtain the following system of equations,

$$
\begin{array}{r}
\Delta \varphi-\frac{1}{c_{p}^{2}} \frac{\partial^{2}}{\partial t^{2}} \varphi+\frac{\left(\varepsilon_{p}-\alpha_{B}\right)}{\lambda+2 \mu} p_{f}=0, \\
\Delta p_{f}-\frac{1}{c_{0}^{2}} \frac{\partial^{2}}{\partial t^{2}} p_{f}+\varepsilon_{p} \rho_{f}\left(\varepsilon_{p}-\alpha_{B}\right) \frac{\partial^{2}}{\partial t^{2}}(\Delta \varphi)=-\frac{\rho_{f}}{\varepsilon_{p}} \frac{\partial^{2}}{\partial t^{2}} s, \\
\frac{1}{c_{s}^{2}} \frac{\partial^{2}}{\partial t^{2}} \mathbf{U}-\Delta \mathbf{U}=0,
\end{array}
$$

where we introduced the following phase velocities

$$
c_{p}=\sqrt{\frac{(\lambda+2 \mu)}{\rho_{d r}}}, \quad c_{s}=\sqrt{\frac{\mu}{\rho_{d r}}} .
$$

At the boundary the Eq. (22) is easily replaced by

$$
\nabla \varphi+\nabla \times \mathbf{U}=0
$$

In dimensionless form, the system (16), (17) and (25) of PDEs is transformed with the following definitions,

$$
\widetilde{\nabla}=\frac{1}{l_{0}} \nabla, \quad \frac{\partial}{\partial t}=\frac{1}{t_{0}} \frac{\partial}{\partial \widetilde{t}}, \quad V=\frac{\phi}{V_{0}}, \quad, \widetilde{\varphi}=\frac{\varphi}{l_{0}^{2}}, \quad, \widetilde{\mathbf{U}}=\frac{\mathbf{U}}{l_{0}^{2}}, \quad \widetilde{p}=\frac{p_{f}}{\bar{p}_{0}}, \quad \widetilde{\mathbf{P}}=\frac{\mathbf{P}}{\overline{\mathbf{P}}_{0}}, \quad \widetilde{s}=\frac{s}{s_{0}} .
$$

The dimensionless system is therefore

$$
\begin{array}{r}
\widetilde{\Delta} \theta+\pi^{2}(\tilde{\nabla} V \cdot \mathbf{n}(\theta))(\widetilde{\nabla} V \cdot * \mathbf{n}(\theta))=0 \\
\widetilde{\nabla} \cdot(\widetilde{\mathbf{P}} \widetilde{\nabla} V)=0 \\
\widetilde{\Delta} \widetilde{p}_{f}-\frac{\partial^{2}}{\partial \widetilde{t}^{2}} \widetilde{p}_{f}+\gamma_{p \phi} \frac{\partial^{2}}{\partial \widetilde{t}^{2}}(\widetilde{\Delta} \widetilde{\varphi})=-\gamma_{p \theta} \frac{\partial^{2}}{\partial \widetilde{t}^{2}} \tilde{s} \\
\widetilde{\Delta} \widetilde{\varphi}-\frac{1}{\tilde{c}_{p}^{2}} \frac{\partial^{2}}{\partial \widetilde{t}^{2}} \widetilde{\varphi}+\gamma_{p \phi} \widetilde{p}_{f}=0 \\
\frac{1}{\tilde{c}_{s}^{2}} \frac{\partial^{2}}{\partial \widetilde{t}^{2}} \widetilde{\mathbf{U}}-\widetilde{\Delta} \widetilde{\mathbf{U}}=0
\end{array}
$$

where

$$
\begin{array}{r}
\phi_{0}=V_{0}=V_{F}=\pi \sqrt{\frac{\kappa_{\mathrm{F}}}{\varepsilon_{a}}}, \quad t_{0}=\frac{l_{0}}{c_{f}}, \quad, l_{0}=\sqrt{t_{0} c_{f}} \quad \widetilde{c}_{p}=\frac{c}{c_{f}} \\
\bar{p}_{0}=c_{f} \sqrt{\varepsilon_{p} \rho_{f}(\lambda+2 \mu)}, \quad \gamma_{p \phi}=\frac{\varepsilon_{p}-\alpha_{B}}{(\lambda+2 \mu)} \bar{p}_{0}, \quad \gamma_{p \theta}=\frac{c_{f} s_{0} \sqrt{\rho_{f}}}{\varepsilon_{p} \sqrt{\varepsilon_{p}(\lambda+2 \mu)}}
\end{array}
$$

\subsection{Plane waves propagating in the porous nematic}

Let us use the plane strain assumption. Thus, the non-dimensional vector potential has only one nonvanishing component, i.e., $\widetilde{\mathbf{U}}=U_{z} \mathbf{e}_{3}$. Considering a plane wave propagating in the $\mathbf{e}_{1}$ direction at radian pulsation $\omega$ and with wavenumber $k$, and setting the source $s=0$, the solution is of the type,

$$
\widetilde{p}_{f}=P_{f} e^{i(\tilde{k} \widetilde{x}-\widetilde{\omega} \tilde{t})}, \quad \widetilde{\varphi}=\Phi e^{i(\widetilde{k} \widetilde{x}-\widetilde{\omega} \widetilde{t})}, \quad U_{z}=W e^{i(\widetilde{k} \widetilde{x}-\widetilde{\omega} \tilde{t})},
$$

where we introduced the following non-dimensional quantities,

$$
\tilde{k}=l_{0} k, \quad \tilde{x}=\frac{x}{l_{0}}, \quad \tilde{\omega}=\omega t_{0} .
$$


Thus, we have the homogeneous system of linear equations, obtained from (29), (30) to (31)

$$
\begin{aligned}
\left(\omega^{2}-k^{2}\right) P_{f}+\omega^{2} k^{2} \gamma_{p \phi} \Phi & =0 \\
\left(\frac{1}{\tilde{c}_{p}^{2}} \omega^{2}-k^{2}\right) \tilde{\phi}+\gamma_{p \phi} \tilde{p}_{f} & =0 \\
\left(-\omega^{2} \frac{1}{\tilde{c}_{s}^{2}}+k^{2}\right) \tilde{U}_{z} & =0
\end{aligned}
$$

The dispersion relations are,

$$
\operatorname{det}\left[\begin{array}{cc}
\left(\omega^{2}-k^{2}\right) & \omega^{2} k^{2} \gamma_{p \phi} \\
\gamma_{p \phi} & \frac{1}{\tilde{c}_{p}^{2}} \omega^{2}-k^{2}
\end{array}\right]=0, \quad-\omega^{2} \frac{1}{\tilde{c}_{s}^{2}}+k^{2}=0
$$

On the one hand, we easily derive that the shear wave velocity is givens by $\widetilde{c}_{s}$. On the other hand, the dispersion relation for pressure waves gives

$$
k^{2}=\frac{1+\tilde{c}_{p}^{2}\left(1+\gamma_{p \phi}^{2}\right) \pm \sqrt{\left(1+\tilde{c}_{p}^{2}+\tilde{c}_{p}^{2} \gamma_{p \phi}^{2}\right)^{2}-4 \omega^{4} \tilde{c}_{p}^{2}}}{2 \tilde{c}_{p}^{2}} .
$$

from which we calculate the following velocities,

$$
\begin{aligned}
& c_{\text {fast }}=\frac{\tilde{c}_{p} \sqrt{2}}{\sqrt{1+\tilde{c}_{p}^{2}\left(1+\gamma_{p \phi}^{2}\right)-\sqrt{1-2 \tilde{c}_{p}^{2}\left(1-\gamma_{p \phi}^{2}\right)+\tilde{c}_{p}^{4}\left(1+\gamma_{p \phi}^{2}\right)^{2}}}}, \\
& c_{\text {slow }}=\frac{\tilde{c}_{p} \sqrt{2}}{\sqrt{1+\tilde{c}_{p}^{2}\left(1+\gamma_{p \phi}^{2}\right)+\sqrt{1-2 \tilde{c}_{p}^{2}\left(1-\gamma_{p \phi}^{2}\right)+\tilde{c}_{p}^{4}\left(1+\gamma_{p \phi}^{2}\right)^{2}}}} .
\end{aligned}
$$

Therefore, we retrieve the classical fast and slow velocities that are well known in the Biot theory. This nonlinear dispersion of NLCs characteristics is due to the micropolar characteristics modeled by the Biot approach. However, it can also be shown by the use of granular materials methods [19].

\section{Numerical simulations}

The object of this section is the presentation of some numerical simulation, with the aim of validating the proposed model with respect to the experiments presented in [3] and to investigate the effects due to porosity. In all the following simulations, we consider as reference configuration for the NLC an electrically unperturbed specimen with spatially constant pressure field. The pressure response of the nematic liquid crystal cell to a voltage input is computed using the finite element method by performing a time-dependent analysis with a BDF solver, always fulfilling the Courant-Friedrichs-Lewy condition. A Fast Fourier Transform (FFT) of the output pressure signal is computed in order to analyze the spectral properties of the response. In both cases we assume that the imposed voltage is larger than the Freedericksz transition threshold: $V>1$.

We will consider the experimental setup presented in Fig. 1. The NLC specimen will be subjected to a time varying voltage applied at the upper electrode, while the lower one is grounded. We will take into account two type of excitations (see Fig. 2): (a) a rectangular pulse and (b) an harmonic signal. In order to estimate the influence of the porous matrix on the wave propagation, we will also consider the specimen without the porous matrix (as in [14]). In order to do so, the coupling parameter $\gamma_{p \phi}$ will be set to zero. 

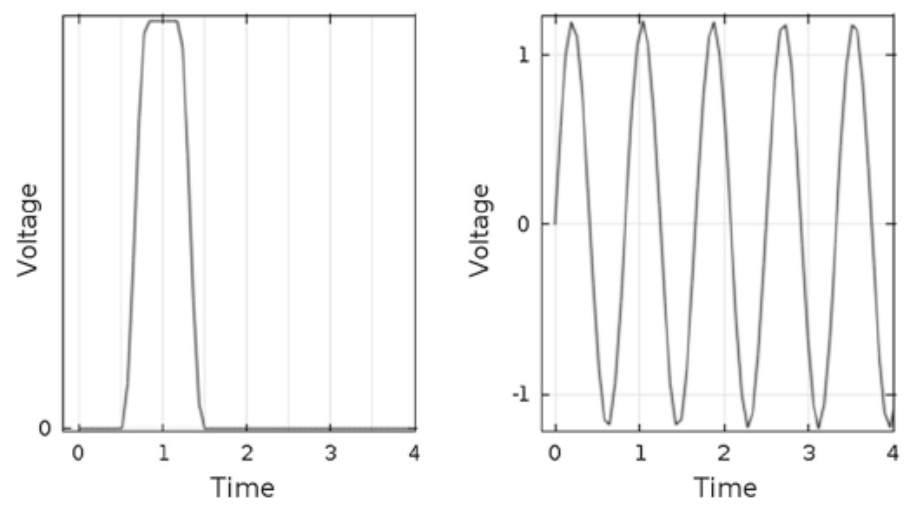

FIG. 2. Shape of the forcing signals

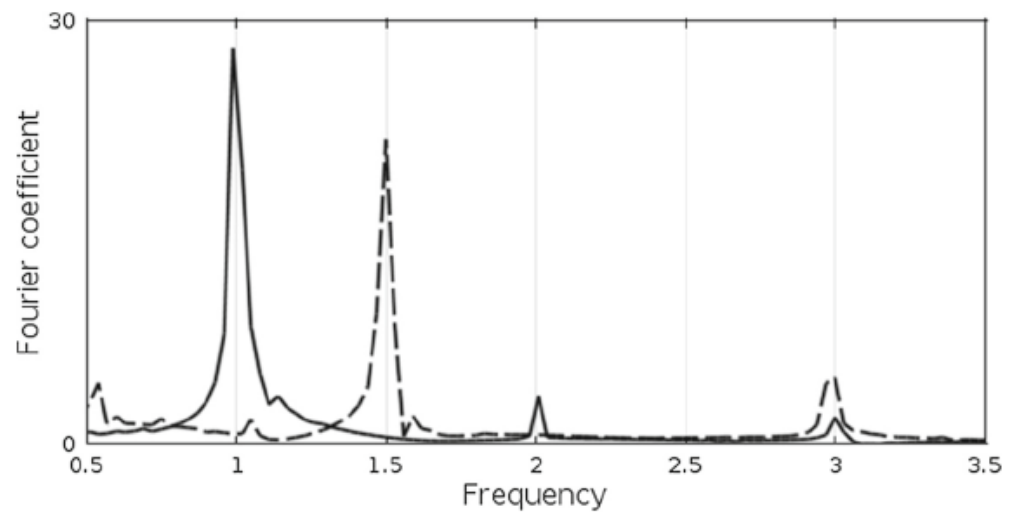

FIG. 3. Fast Fourier transforms of the pressure signal for a pulse excitation in the case of $\gamma_{p \phi}=0.5$ (dashed line) and $\gamma_{p \phi}=0$ (solid line)

We start by analyzing the case of a pulse excitation, as in the left side of Fig. 2. The aim is to have evidence of the acoustic generation in the nematic cell and to evaluate the effects due to the presence of the porous matrix on the resonance frequencies of the cell.

In Fig. 3 the FFT of the pressure field in a point of the NLC is plotted. Let us consider the case of vanishing poroelastic coupling first, i.e., solid line $\left(\gamma_{p \phi}=0\right)$. As it can be verified, an acoustic signal is generated, and one can easily identify the resonance frequencies of the specimen, $\tilde{f}=(1,2,3, \ldots)$. When considering a non-vanishing poroelastic coupling, i.e., $\gamma_{p \phi} \neq 0$. (dashed line in Fig. 3), these resonance frequencies are shifted, and are equal to $\tilde{f}=(1.5,3, \ldots)$.

In the second simulation, we consider the response to an harmonic voltage signal $V=1.2 \sin (2 \pi \times 1.2 \tilde{t})$ having a non-dimensional frequency $\tilde{f}=\tilde{f}_{h}=1.2$, as plotted in the right side of Fig. 2 . The corresponding FFT is pictured in Fig. 4. Let us start again from the analysis of a vanishing poroelastic coupling coefficient (solid line). First of all we can observe the resonance frequencies of the cell are still excited. This is due to the nonlinearities of the nemato-acoustic coupling, which behaves as a rectifier of the signal (details can be found in Sect. 3.1). As a consequence of the same rectifying behavior, we can observe that the frequency of excitation $\tilde{f}_{h}$ is doubled in the spectrum of the response. The same considerations can be done in the case of non-vanishing poroelastic coefficient, where we can also observe a low frequency peak due to the resonance frequency of the cell, modified by the effect of the poroelastic coupling. 


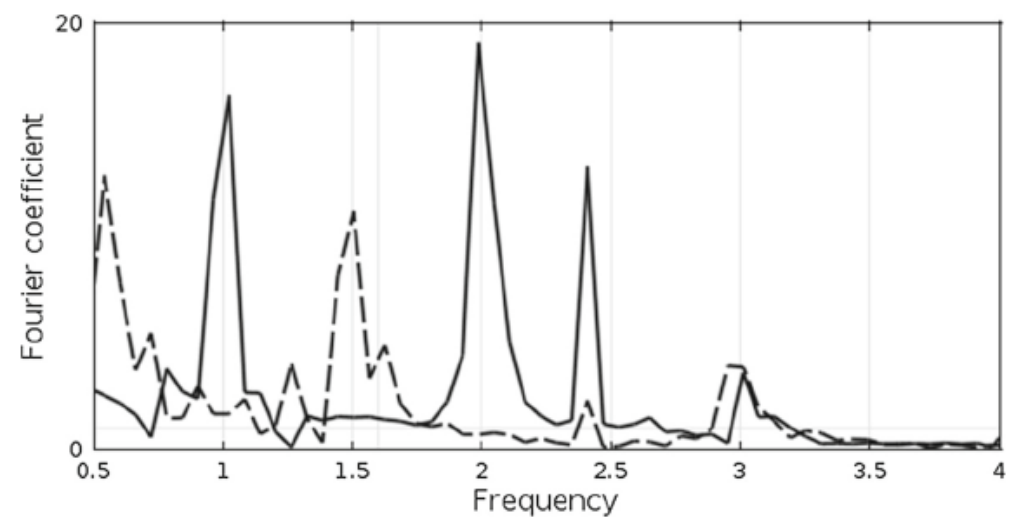

FIG. 4. Fast Fourier transforms of the pressure signal for an harmonic excitation of frequency $\tilde{f}=1.2$ in the case of $\gamma_{p \phi}=0.5$ (dashed line) and $\gamma_{p \phi}=0$ (solid line)

\subsection{Some considerations on the nature of the forcing term and its relationship with Freedrisksz phase transition}

As it is well known from the literature, see e.g. [1,15], the term which governs the behavior of nematic liquid crystals, in our case Eqs. (16) and (17), has a strong nonlinear character. In particular, when considering a thin cell and when imposing a suitable external electric field, a pitchfork supercritical bifurcation, called Freedrisksz phase transition, is observed. For a thin cell with strong anchoring condition, i.e., and imposed value of $\theta$ at the boundary, and for a vertical external electric field, the value of the threshold is

or

$$
V_{F}=\pi \sqrt{\frac{\kappa_{\mathrm{F}}}{\varepsilon_{a}}}
$$

$$
E=\frac{\pi}{d} \sqrt{\frac{\kappa_{\mathrm{F}}}{\varepsilon_{a}}}
$$

Indeed, when considering time varying electric fields which are able to cross the bifurcation, the NLC cell acts as a nonlinear rectifier. This rectifying behavior is responsible for the appearance of peaks at twice the frequency of excitation in the case of an harmonic excitation 4. It is also worth remarking that an electric signal with amplitude lower than $V_{F}$ will not generate any perturbation in the nematic field. This behavior has been observed experimentally, again by [3].

\section{Conclusions and perspectives}

The researches presented in $[14,20]$ and in this paper were motivated by some experimental observations [3]. Besides, the possibility of developing a new concept of nematic cell in which the mechanical interaction between different nematicons can be damped via a Darcy or Brinkman, or Stokes-Navier type friction motivated the presented mathematical modeling. In the conceived poro-nematic cells, the nematic liquid mass density is changed by means of distortion of the nematic field, thus causing a pressure and velocity fields time variation. The friction phenomena thus excited are assumed to be able to decrease the mechanical range of influence around a solitary excitation of the nematic liquid crystal.

Differently from what done by aforementioned authors, in this work, by following [14] we have adopted a model for a compressible NLC in which the spatial gradient of the director field $\mathbf{n}$ is coupled with the 
mass density $\varrho_{f}$. However, this model is still not complete, as it does not catch completely all acousticnematic coupling phenomena. Indeed, in order to account for the phenomena observed by Selinger [8], one should generalize the constitutive relation between $p_{f}, \varrho_{f}, \mathbf{n}$ and $\nabla \mathbf{n}$ introduced in the present paper. This generalization will be subject of future investigations, that will be based on generalized continuum theories, where a more complete treatment will be found by a postulation based on Hamilton-Rayleigh principle.

The presented numerical simulations seem to support the concept underlying the proposed model and further investigations, both theoretical and experimental, seem justified. From this point of view, an investigation on proper finite element (FE) simulation [21-29] or regularized FE [30-37] will be welcome in order to avoid, e.g., the unpleasant occurrence of instability [38-49]. Due to the high porosity, the Biot model should probably be modified, see e.g. [50-58], by adding a correction term which takes into account the microstructural effect [48,59-64]. Strain gradient models (see e.g. [2,65] for classical references and $[66,67]$ for more recent results) are good candidates for this purpose, as shown in [68-70]. A review of results on the theoretical foundation of a variational approach for higher gradient theories is [71]. Besides, the use of the methods of metamaterials [72-74] like those for pantographic structures [75-77] could be considered even to take into account the effects of damage [78-82]. Another possible extension of this model is to include surface effects [83-89] and the anisotropy induced by the orientation of the NLC directors, as in [90], or as it is done in granular materials [91].

\section{Acknowledgements}

We are grateful to professor Antonio Di Carlo for the fruitful discussions about the subject of this paper. Giuseppe Rosi wish to thank the Faculté des Sciences et Technologies of The Université Paris-Est Créteil for the financial support. The authors finally acknowledge the Laboratoire international associé LIA Coss \& Vita for the financial support.

\section{References}

[1] de Gennes, P.G., Prost, J.: The Physics of Liquid Crystals, 2nd edn. Oxford University Press, Oxford (1995)

[2] Eringen, A.C.: Microcontinuum Field Theories I: Foundations and Solids. Springer, Berlin (1999)

[3] Kim, Y., Patel, J.: Acoustic generation in liquid crystals. Appl. Phys. Lett. 75, 1985 (1999)

[4] Greanya, V., Malanoski, A., Weslowski, B., Spector, M., Selinger, J.: Dynamics of the acousto-optic effect in a nematic liquid crystal. Liq. Cryst. 32(7), 933-941 (2005)

[5] Greanya, V., Spector, M., Selinger, J., Weslowski, B., Shashidhar, R.: Acousto-optic response of nematic liquid crystals. J. Appl. Phys. 94(12), 7571-7575 (2003)

[6] Malanoski, A., Greanya, V., Weslowski, B., Spector, M., Selinger, J., Shashidhar, R.: Theory of the acoustic realignment of nematic liquid crystals. Phys. Rev. E 69(2), 021705 (2004)

[7] Satiro, C., Vitoriano, C.: Director fluctuations in nematic liquid crystals induced by an ultrasonic wave. Phys. Rev. E 86(1), 011701 (2012)

[8] Selinger, J., Spector, M., Greanya, V., Weslowski, B., Shenoy, D., Shashidhar, R.: Acoustic realignment of nematic liquid crystals. Phys. Rev. E 66(5), 051708 (2002)

[9] Challamel, N., Wang, C.M.: The small length scale effect for a non-local cantilever beam: a paradox solved. Nanotechnology 19(34), 345703 (2008)

[10] Challamel, N., Rakotomanana, L., Le Marrec, L.: A dispersive wave equation using nonlocal elasticity. Comptes Rendus Mécanique 337(8), 591-595 (2009)

[11] dell'Isola, F., Seppecher, P.: Edge contact forces and quasi-balanced power. Meccanica 32(1), 33-52 (1997)

[12] de Matteis, G., Virga, E.G.: Director libration in nematoacoustics. Phys. Rev. E 83(1), 001703 (2011)

[13] Virga, E.G.: Variational theory for nematoacoustics. Phys. Rev. E 80(3), 031705 (2009)

[14] Rosi, G., Teresi, L., dell'Isola, F., DiCarlo, A.: Coupling between mass density and director arrangement in nematic liquid crystals. In: European Solid Mechanics Conference 2012, pp. 1-2, July 2012

[15] Khoo, I.-C.: Liquid Crystals. Wiley-Blackwell, Hoboken (2007) 
[16] Biot, M.A.: Theory of propagation of elastic waves in a fluid saturated porous solid. I. Low frequency range. J. Acoust. Soc. Am. 28, 168 (1956)

[17] Biot, M.A.: Theory of propagation of elastic waves in a fluid saturated porous solid. II. Higher frequency range. J. Acoust. Soc. Am. 28(2), 179-191 (1956)

[18] Biot, M.A.: Mechanics of deformation and acoustic propagation in porous media. J. Appl. Phys. 33(4), 1482-1498 (1962)

[19] Misra, A., Poorsolhjouy, P.: Granular micromechanics based micromorphic model predicts frequency band gaps. Continuum Mech. Thermodyn. 28, 215-234 (2016)

[20] Rosi, G., Pouget, J., dell'Isola, F.: Control of sound radiation and transmission by a piezoelectric plate with an optimized resistive electrode. Eur. J. Mech. A-Solids 29(5), 859-870 (2010)

[21] Baraldi, D., Reccia, E., Cazzani, A., Cecchi, A.: Comparative analysis of numerical discrete and finite element models: the case of in-plane loaded periodic brickwork. Compos. Mech. Comput. Appl. 4(4), 319-344 (2013)

[22] Bilotta, A., Turco, E.: A numerical study on the solution of the Cauchy problem in elasticity. Int. J. Solids Struct. 46(25-26), 4451-4477 (2009)

[23] Cazzani, A., Ruge, P.: Numerical aspects of coupling strongly frequency-dependent soil-foundation models with structural finite elements in the time-domain. Soil Dyn. Earthq. Eng. 37, 56-72 (2012)

[24] Garusi, E., Tralli, A., Cazzani, A.: An unsymmetric stress formulation for reissner-mindlin plates: a simple and lockingfree rectangular element. Int. J. Comput. Mater. Sci. Eng. 5(3), 589-618 (2004)

[25] Greco, L., Cuomo, M.: Consistent tangent operator for an exact Kirchhoff rod model. Continuum Mech. Thermodyn. 27(4-5), 861-877 (2015)

[26] Presta, F., Hendy, C.R., Turco, E.: Numerical validation of simplified theories for design rules of transversely stiffened plate girders. Struct. Eng. 86(21), 37-46 (2008)

[27] Cazzani, A.: On the true extrema of Youngs modulus in hexagonal materials. Appl. Math. Comput. 238, 397-407 (2014)

[28] Cazzani, A., Ruge, P.: Symmetric matrix-valued transmitting boundary formulation in the time-domain for soil-structure interaction problems. Soil Dyn. Earthq. Eng. 57, 104-120 (2014)

[29] Cazzani, A., Ruge, P.: Stabilization by deflation for sparse dynamical systems without loss of sparsity. Mech. Syst. Signal Process. 70-71, 664-681 (2016)

[30] Greco, L., Cuomo, M.: An implicit G1 multi patch B-spline interpolation for Kirchhoff-Love space rod. Comput. Methods Appl. Mech. Eng. 269, 173-197 (2014)

[31] Greco, L., Cuomo, M.: An isogeometric implicit G1 mixed finite element for Kirchhoff space rods. Comput. Methods Appl. Mech. Eng. 298, 325-349 (2016)

[32] Cuomo, M., Contrafatto, L., Greco, L.: A variational model based on isogeometric interpolation for the analysis of cracked bodies. Int. J. Eng. Sci. 80, 173-188 (2014)

[33] Cazzani, A., Malagò, M., Turco, E.: Isogeometric analysis of plane-curved beams. Math. Mech. Solids 28(1-2), 139-156 (2016)

[34] Cazzani, A., Malagò, M., Turco, E.: Isogeometric analysis: a powerful numerical tool for the elastic analysis of historical masonry arches. Continuum Mech. Thermodyn. 28(1-2), 139-156 (2016)

[35] Bilotta, A., Formica, G., Turco, E.: Performance of a high? Continuity finite element in threedimensional elasticity. Int. J. Numer. Methods Biomed. Eng. 26(9), 1155-1175 (2010)

[36] Alessandrini, G., Bilotta, A., Morassi, A., Rosset, E., Turco, E.: Computing volume bounds of inclusions by EIT measurements. J. Sci. Comput. 33(3), 293-312 (2007)

[37] Alessandrini, G., Bilotta, A., Formica, G., Morassi, A., Rosset, E., Turco, E.: Numerical size estimates of inclusions in elastic bodies. Inverse Probl. 21(1), 133-151 (2005)

[38] Rizzi, N.L., Varano, V.: The effects of warping on the postbuckling behaviour of thin-walled structures. Thin-Walled Struct. 49(9), 1091-1097 (2011)

[39] Ruta, G.C., Varano, V., Pignataro, M., Rizzi, N.L.: A beam model for the flexural-torsional buckling of thin-walled members with some applications. Thin-Walled Struct. 46(7), 816-822 (2008)

[40] AminPour, H., Rizzi, N.: A one-dimensional continuum with microstructure for single-wall carbon nanotubes bifurcation analysis. Math. Mech. Solids 21(2), 168-181 (2016)

[41] Luongo, A., Piccardo, G.: Linear instability mechanisms for coupled translational galloping. J. Sound Vib. 288(4), 1027-1047 (2005)

[42] Piccardo, G., Pagnini, L.C., Tubino, F.: Some research perspectives in galloping phenomena: critical conditions and post-critical behavior. Contin. Mech. Thermodyn. 27(1-2), 261-285 (2015)

[43] Luongo, A., Piccardo, G.: A continuous approach to the aeroelastic stability of suspended cables in 1: 2 internal resonance. J. Vib. Control 14(1-2), 135-157 (2008)

[44] Luongo, A., D'Annibale, F.: Double zero bifurcation of non-linear viscoelastic beams under conservative and nonconservative loads. Int. J. Non-Linear Mech. 55, 128-139 (2013)

[45] Luongo, A., D'annibale, F.: Bifurcation analysis of damped visco-elastic planar beams under simultaneous gravitational and follower forces. Int. J. Mod. Phys. B 26(25), 1246015 (2012) 
[46] Rizzi, N.L., Varano, V., Gabriele, S.: Initial postbuckling behavior of thin-walled frames under mode interaction. ThinWalled Struct. 68, 124-134 (2013)

[47] Bersani, A.M., Giorgio, I., Tomassetti, G.: Buckling of an elastic hemispherical shell with an obstacle. Continuum Mech. Thermodyn. 25(2-4), 443-467 (2013)

[48] Cecchi, Antonella, Rizzi, Nicola L.: Heterogeneous elastic solids: a mixed homogenization-rigidification technique. Int. J. Solids Struct. 38(1), 29-36 (2001)

[49] Misra, A., Poorsolhjouy, P.: Micro-macro scale instability in 2D regular granular assemblies. Continuum Mech. Thermodyn. 27(1-2), 63-82 (2013)

[50] Yang, Y., Ching, W.Y., Misra, A.: Higher-order continuum theory applied to fracture simulation of nanoscale intergranular glassy film. J. Nanomech. Micromech. 1(2), 60-71 (2011)

[51] Goda, I., Assidi, M., Ganghoffer, J.F.: A 3D elastic micropolar model of vertebral trabecular bone from lattice homogenization of the bone microstructure. Biomech. Model Mechanobiol. 13(1), 53-83 (2014)

[52] dell'Isola, F., Guarascio, M., Hutter, K.: A variational approach for the deformation of a saturated porous solid. A second-gradient theory extending Terzaghi's effective stress principle. Arch. Appl. Mech. 70(5), 323-337 (2000)

[53] Yang, Y., Misra, A.: Micromechanics based second gradient continuum theory for shear band modeling in cohesive granular materials following damage elasticity. Int. J. Solids Struct. 49(18), 2500-2514 (2012)

[54] Andreaus, U., Giorgio, I., Lekszycki, T.: A 2D continuum model of a mixture of bone tissue and bio? Resorbable material for simulating mass density redistribution under load slowly variable in time. ZAMM J. Appl. Math. Mech./Zeitschrift für Angewandte Mathematik und Mechanik 94(12), 978-1000 (2014)

[55] Andreaus, U., Colloca, M., Iacoviello, D.: An optimal control procedure for bone adaptation under mechanical stimulus. Control Eng. Pract. 20(6), 575-583 (2012)

[56] Giorgio, I., Andreaus, U., Lekszycki, T., Della, Corte A.: The influence of different geometries of matrix/scaffold on the remodeling process of a bone and bio-resorbable material mixture with voids. Math. Mech. Solids (2015). doi:10.1177/ 1081286515616052

[57] Giorgio, I., Andreaus, U., Scerrato, D., Braidotti, P.: Modeling of a non-local stimulus for bone remodeling process under cyclic load: application to a dental implant using a bioresorbable porous material. Math. Mech. Solids (2016). doi: $10.1177 / 1081286516644867$

[58] Andreaus, U., Colloca, M., Iacoviello, D.: Optimal bone density distributions: numerical analysis of the osteocyte spatial influence in bone remodeling. Comput. Methods Progr. Biomed. 113(1), 80-91 (2014)

[59] Piccardo, G., Ranzi, G., Luongo, A.: A complete dynamic approach to the generalized beam theory cross-section analysis including extension and shear modes. Math. Mech. Solids 19(8), 900-924 (2014)

[60] Piccardo, G., Ranzi, G., Luongo, A.: A direct approach for the evaluation of the conventional modes within the GBT formulation. Thin-Walled Struct. 74, 133-145 (2014)

[61] Piccardo, G., D'Annibale, F., Zulli, D.: On the contribution of Angelo Luongo to Mechanics: in honor of his 60th birthday. Continuum Mech. Thermodyn. 27(4-5), 507-529 (2015)

[62] Luongo, A., Zulli, D.: A non-linear one-dimensional model of cross-deformable tubular beam. Int. J. Non Linear Mech. 66, 33-42 (2014)

[63] Selvadurai, P.A., Selvadurai, A.P.S.: On the effective permeability of a heterogeneous porous medium: the role of the geometric mean. Philos. Mag. 94(20), 2318-2338 (2014)

[64] dell'Isola, F., Andreaus, U., Placidi, L.: At the origins and in the vanguard of peridynamics, non-local and higher gradient continuum mechanics. An underestimated and still topical contribution of Gabrio Piola. Math. Mech. Solids 20, 887-928 (2015)

[65] Mindlin, R.D., Mindlin, R.D.: Micro-structure in linear elasticity. Arch. Ration. Mech. Anal. 16(1), 51-78 (1964)

[66] Alibert, J.J., Della Corte, A.: Second-gradient continua as homogenized limit of pantographic microstructured plates: a rigorous proof. Zeitschrift für angewandte Mathematik und Physik 66(5), 2855-2870 (2015)

[67] Enakoutsa, K., Della Corte, A., Giorgio, I.: A model for elastic flexoelectric materials including strain gradient effects. Math. Mech. Solids, 1081286515588638 (2015)

[68] Rosi, G., Madeo, A., Guyader, J.: Switch between fast and slow Biot compression waves induced by "second gradient microstructure" at material discontinuity surfaces in porous media. Int. J. Solids Struct. 50(10), 1721-1746 (2013)

[69] Sciarra, G., dell'Isola, F., Coussy, O.: Second gradient poromechanics. Int. J. Solids Struct. 44(20), 6607-6629 (2007)

[70] Sciarra, G., dell'Isola, F., Ianiro, N., Madeo, A.: A variational deduction of second gradient poroelasticity I: general theory. J. Mech. Mater. Struct. 3(3), 507-526 (2008)

[71] dell'Isola, F., Seppecher, P., Della Corte, A.: The postulations á la D? Alembert and á la Cauchy for higher gradient continuum theories are equivalent: a review of existing results. In: Proceedings of the Royal Society A, vol. 471, No. 2183, p. 20150415. The Royal Society (2015)

[72] Alibert, J.J., Seppecher, P., dell'Isola, F.: Truss modular beams with deformation energy depending on higher displacement gradients. Math. Mech. Solids 8(1), 51-73 (2003)

[73] Del Vescovo, D., Giorgio, I.: Dynamic problems for metamaterials: review of existing models and ideas for further research. Int. J. Eng. Sci. 80, 153-172 (2014) 
[74] Giorgio, I., Galantucci, L., Della, Corte A., Del Vescovo, D.: Piezo-electromechanical Smart Materials with distributed arrays of Piezoelectric Transducers: current and upcoming applications. Int. J. Appl. Electromagn. Mech. 47(4), 10511084 (2015)

[75] dell'Isola, F., Lekszycki, T., Pawlikowski, M., Grygoruk, R., Greco, L.: Designing a light fabric metamaterial being highly macroscopically tough under directional extension: first experimental evidence. Zeitschrift für angewandte Mathematik und Physik 66(6), 3473-3498 (2015)

[76] Rahali, Y., Giorgio, I., Ganghoffer, J.F., dell'Isola, F.: Homogenization a la Piola produces second gradient continuum models for linear pantographic lattices. Int. J. Eng. Sci. 97, 148-172 (2015)

[77] Scerrato, D., Giorgio, I., Rizzi, N.L.: Three-dimensional instabilities of pantographic sheets with parabolic lattices: numerical investigations. ZAMP - Zeitschrift für angewandte Mathematik und Physik/J. Appl. Math. Phys. (2016). doi:10.1007/s00033-016-0650-2

[78] Del Vescovo, D., Fregolent, A.: Theoretical and experimental dynamic analysis aimed at the improvement of an acoustic method for fresco detachment diagnosis. Mech. Syst. Signal Process. 23(7), 2312-2319 (2009)

[79] Misra, A., Singh, V.: Thermomechanics-based nonlinear rate-dependent coupled damage-plasticity granular micromechanics model. Continuum Mech. Thermodyn. 27(4), 787-817 (2015)

[80] Roveri, N., Carcaterra, A.: Damage detection in structures under traveling loads by Hilbert-Huang transform. Mech. Syst. Signal Process. 28, 128-144 (2012)

[81] Andreaus, U., Baragatti, P.: Cracked beam identification by numerically analysing the nonlinear behaviour of the harmonically forced response. J. Sound Vib. 330(4), 721-742 (2011)

[82] Andreaus, U., Ceradini, G., D'Asdia, P., Gaudenzi, P.: Damage modelling and seismic response of simple degrading systems. Res Mech. 22(1), 79-100 (1987)

[83] Naumenko, K., Eremeyev, V.A.: A layer-wise theory for laminated glass and photovoltaic panels. Compos. Struct. 112(1), 283-291 (2014)

[84] Rosi, G., Giorgio, I., Eremeyev, V.A.: Propagation of linear compression waves through plane interfacial layers and mass adsorption in second gradient fluids. ZAMM Zeitschrift fur Angewandte Mathematik und Mechanik 93(12), 914-927 (2013)

[85] Steigmann, D.J., Pipkin, A.C.: Wrinkling of pressurized membranes. J. Appl. Mech. 56(3), 624-628 (1989)

[86] Steigmann, D.J., Pipkin, A.C.: Finite deformations of wrinkled membranes. Q. J. Mech. Appl. Math. 42(3), 427-440 (1989)

[87] Altenbach, H., Eremeev, V.A., Morozov, N.F.: On equations of the linear theory of shells with surface stresses taken into account. Mech. Solids 45(3), 331-342 (2010)

[88] Misra, A., Huang, S.: Micromechanical stress-displacement model for rough interfaces: effect of asperity contact orientation on closure and shear behavior. Int. J. Solids Struct. 49(1), 111-120 (2012)

[89] Berezovski, A., Giorgio, I., Della, Corte A.: Interfaces in micromorphic materials: wave transmission and reflection with numerical simulations. Math. Mech. Solids 21(1), 37-51 (2016)

[90] Biscari, P., DiCarlo, A., Turzi, S.S.: Anisotropic wave propagation in nematic liquid crystals. Soft. Matter. 10(41), 8296-8307 (2014)

[91] Misra, A., Poorsolhjouy, P.: Granular micromechanics model of anisotropic elasticity derived from Gibbs potential. Acta Mech. 227(5), 1393-1413 (2016)

Giuseppe Rosi, Luca Placidi and Francesco dell'Isola

International Telematic University Uninettuno

Roma

Italy

e-mail: luca.placidi@uninettunouniversity.net 